the

abdus salam

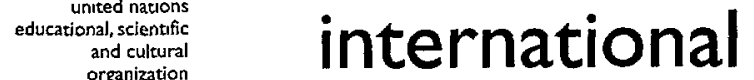

centre



physics

QUALITY DESCRIPTORS OF OPTICAL BEAMS BASED ON CENTRED REDUCED MOMENTS I: SPOT ANALYSIS

\author{
Román Castañeda \\ Jorge Garcia-Sucerquia \\ and
}

Frank Brand 
United Nations Educational Scientific and Cultural Organization and

International Atomic Energy Agency

THE ABDUS SALAM INTERNATIONAL CENTRE FOR THEORETICAL PHYSICS

\title{
QUALITY DESCRIPTORS OF OPTICAL BEAMS BASED ON CENTRED REDUCED MOMENTS I: SPOT ANALYSIS
}

\author{
Román Castañeda ${ }^{1}$ \\ Physics Department, Universidad Nacional de Colombia Sede Medellín, \\ A.A. 3840 Medellín, Colombia \\ and \\ The Abdus Salam International Centre for Theoretical Physics, Trieste, Italy, \\ Jorge Garcia-Sucerquia ${ }^{2}$ \\ Physics Department, Universidad Nacional de Colombia Sede Medellín, \\ A.A. 3840 Medellín, Colombia \\ and \\ Frank Brand ${ }^{3}$ \\ Berlin School of Economics, Badensche Str. 50-51, 10825 Berlin, Germany. \\ MIRAMARE - TRIESTE \\ March 2003
}

\footnotetext{
${ }^{1}$ Regular Associate of the Abdus Salam ICTP. rcastane@perseus.unalmed.edu.co

2 jigarcia@ perseus.unalmed.edu.co

3 frank.brand@t-online.de
} 


\begin{abstract}
A method for analyzing beam spots is discussed. It is based on the central reduced moments of the spot and its associated density functions. These functions allow us to separately analyze specific spot fractions, in such a way that specific combinations of higher order moments can be interpreted as coordinates of their centre of mass and the length and orientations of their principal axis. So, the descriptors of the associated density functions deal with the quantitative estimation of spot features, such as coma-like and astigmatism-like distortions.
\end{abstract}

To assure high accuracy, background noise suppression and an optimal match of the spot support onto the region $[-1,1] \times[-1,1]$ are performed prior to the calculation of the moments. Simulations were performed for illustrating the method.

\title{
1. INTRODUCTION
}

The definition of quality descriptors and the procedures for their determination are crucial for the quality assessment of optical beams [1,2]. To this aim, the moments of the Wigner Distribution Function (WDF), which describe optical fields in any state of spatial coherence [3], are growing in importance [4-8].

The existence of geometrical moments for any type of beam profiles is definitive for the development of general applications. In particular, the moments of order higher than the second of hard-edge diffracted beams will diverge, in such a way that the quality descriptors cannot be defined. However, some solutions to this limitation have been yet reported [4].

In such a way, from a general point of view low order moments of the WDF specify the optical axis and the principal axes of the field distribution. They are determined by setting to zero the first-order moments and the cross second-order moment respectively. Once the principal axes are determined, the second-order moments will be a measure of the width and the angular divergence of the beam along these axes [1].

Second-order moments of the WDF allow defining the beam quality factor $Q$, which takes its minimum value for a Gaussian beam. So, the ratio between the $Q$ value of an arbitrary beam and that for a Gaussian one, usually denoted by $M^{2}$, is a measure of the relative far-field spreading of the arbitrary beam relative to an ideal Gaussian beam with the same waist [1].

Third-order moments account for the symmetry of the field distribution and the spatial range of the beam's symmetry; and fourth-order moments yield to the definition of the kurtosis, which provides a quantitative measure for beam classification according to their sharpness. A one-dimensional beam is leptokurtic, mesokurtic or platykurtic if this parameter is higher, equal or lower than 3 respectively, which is the value for a pure one-dimensional Gaussian beam [1]. Higher order moments are also used for describing fine details of the field distribution, but their interpretation is usually difficult.

Now, we want to discuss alternative procedures to determine quality descriptors of optical beams based on centered reduced moments. In this paper we mainly concern with the characterization of beam spots, i.e. the power distribution of the beam on a plane orthogonal to its propagation axis. This quantity can be obtained by evaluating the corresponding WDF at individual points of the plane $[9,10]$. 
With this aim, we introduce the associated density functions [11-13], whose first and second order moments can be expressed as combinations of the higher order moments of the spot power distribution. So, a well-defined interpretation can be done to these combinations instead of individual moments, in terms of coordinates of the centre of mass of the associated density functions and the length and orientation of these main axes. This interpretation deals to quantitative descriptors of the spot features.

\section{CENTRED REDUCED MOMENTS}

The geometrical moments of a two-dimensional density function $\rho\left(x^{\prime}, y^{\prime}\right)$ are defined as [11-13]

$$
M_{m n}=\int_{-\infty}^{\infty} \int_{-\infty}^{\infty} x^{\prime m} y^{\prime n} \rho\left(x^{\prime}, y^{\prime}\right) d x^{\prime} d y^{\prime}
$$

with $m, n=0,1,2, \ldots$ There are $m+n+1$ moments of order $m+n$. The normalization of the density function is described by its moment of zero-order, i.e. $M_{00}=1$, and the coordinates of its centre of mass by the first order moments, i.e. $\left\langle x^{\prime}\right\rangle=M_{10}$ and $\left\langle y^{\prime}\right\rangle=M_{01}$.

It is usual to refer the higher-order moments to the centre of mass of the density function by applying the shift transformation $x^{\prime} \rightarrow x^{\prime}-\left\langle x^{\prime}\right\rangle$ and $y^{\prime} \rightarrow y^{\prime}-\left\langle y^{\prime}\right\rangle$. As a consequence, the firstorder moments are set to zero and the higher-order moments will be called centred moments [1113].

Density functions of practical interest (i.e. normalized power distributions of a beam spot) usually decay in such a way that their significant values are taken inside a finite region, which is called the support of the density function. It is essentially determined by the detector noise in many applications, because the very low values of the density function outside its support will be always smaller than the noise level of the detector and cannot be recorded. Thus, the support edge can be determined by locally analysing the signal-to-noise ratio of the recorded power distribution.

In general, $\rho(x, y)$ can be considered as a function of finite support and its values outside the support can be set to zero.

Reduced moments are useful for describing density functions with finite support. They are obtained by scaling the $\left(x^{\prime}, y^{\prime}\right)$ coordinates to the reduced coordinates $x=\frac{x^{\prime}}{A}$ and $y=\frac{y^{\prime}}{B}$, with $2 A$ and $2 B$ the side lengths of the support along the $x$ - and the $y$-axis respectively. Thus, equation (1) becomes $M_{m n}=A^{m+1} B^{n+1} \gamma_{m n}$, with

$$
\gamma_{m n}=\int_{-1}^{1} \int_{-1}^{1} x^{m} y^{n} \rho(x, y) d x d y
$$

the reduced moments of the two dimensional density function $\rho(x, y)$ [11-13]. They will be called centred reduced moments if they are also referred to the centre of mass of the density function. It is 
apparent from equation (2) that all odd-order centred reduced moments will be equal to null only if $\rho(x, y)$ exhibits even-symmetry (i.e. rotation symmetry). Furthermore, $\gamma_{j k}=\gamma_{k j}$ for $j, k=0,2,4, \ldots$ in this case.

Now, recording procedures usually provide additive noise, which must be suppressed for properly determining the moments. The noisy density function takes the form $\rho(x, y)+n(x, y)$, with $n(x, y)$ the density function of noise. Because of the shape of the density functions, noise often is dominant in the vicinity of the support edge, where the factor $x^{m} y^{n}$ provides the most important enhancement. Furthermore, a region of important values of $\rho(x, y)$ around the coordinate origin (its centre of mass) is screened by the small values of $x^{m} y^{n}$ there, in such a way that the higher the moments order the greater the size of this region. As a consequence, the noise impact on the calculation of the moments significantly increases with the order of the moments. Therefore, noise suppression is a essential issue for an accurate determination of the moments, as we discuss below.

\section{CHARACTERISATION OF A BEAM SPOT}

Let us consider the power distribution at the frequency $\omega$ of the beam spot at the plane $P$, i.e. $S_{P}(u, v ; \omega)$. The function

$$
\rho_{P}(x, y ; \omega)=\frac{S_{P}(x, y ; \omega)}{\iint_{P} S_{P}(x, y ; \omega) d x d y}
$$

fulfils the axioms of the two dimensional density functions, i.e. it is a normalized and positive definite function, which decay to null outside a finite region around the propagation axis. So, $\rho_{P}(x, y ; \omega)$ is the density function related to the considered beam spot, whose reduced moments will be obtained by replacing them into equation (2) after the appropriate scaling of its support.

As expected, $\gamma_{00}=1$ and the first-order moments determine the position of the centre of mass of the spot, i.e. $[\langle x\rangle,\langle y\rangle] \leftrightarrow\left(\gamma_{10}, \gamma_{01}\right)$. The remaining spot features are determined by recalculating the moments with respect to the centre of mass of the spot, i.e. by replacing $x^{m} \rightarrow\left(x-\gamma_{10}\right)^{m}$ and $y^{n} \rightarrow\left(y-\gamma_{01}\right)^{n}$ with $m, n>1$ in the integral of moments. Thus, these quantities will be the centred reduced moments of the spot. In the following we will refer only to this type of moments by assuming their coordinates implicitly centred and reduced.

The principal axes of the beam spot and its lengths will be specified by the second order moments, after setting $\gamma_{11}$ to zero by properly rotating the coordinate axes on the plane by the angle [11-13]

$$
\theta_{P}=\frac{1}{2} \arctan \left[\frac{\gamma_{11}}{\gamma_{20}-\gamma_{02}}\right]
$$


Indeed, $a=\sqrt{\gamma_{20}^{\prime}}$ and $b=\sqrt{\gamma_{02}^{\prime}}$ will be the lengths of the principal axes of the beam spot, with the primes denoting the values of the moments after the rotation of the coordinate axes.

Now, the spot eccentricity is a descriptor of stigmatism of the beam. It is given by $E_{P}=\sqrt{1-\left(\frac{a}{b}\right)^{2}}$ provided $a \leq b$. So, the spot will be ideally stigmatic (i.e. circular symmetric) for $a=b$ and astigmatic otherwise. However, regarding a departure of $5 \%$ between the lengths of the principal axes as permissible, we will have $0 \leq E_{p} \leq 0.3$ for stigmatic spots and $E_{p}>0.3$ for astigmatic ones.

The four moments of third order are related to the symmetry of the corresponding spot around its centre of mass, and the five of fourth order describe the kurtosis of the spot. Although higher order moments are related to finer detail of the spots, their interpretation as descriptors of specific features is usually difficult.

However, it is possible to overcome this limitation by introducing associated density functions [1113], which are defined as

$$
V_{P}^{(q)}(x, y ; \omega)=C_{q}\left(x^{2}+y^{2}\right)^{q} \rho_{P}(x, y ; \omega)
$$

with $q=0,1,2 \ldots$, and $C_{q}$ a normalization constant. Table 1 shows the associated density functions up to the fifth-order. In these functions it is apparent that $C_{q}^{-1}$ results from a proper linear combination of $\gamma$-moments of $2 q$-order.

The factor $\left(x^{2}+y^{2}\right)^{q}$ denote a set of revolution surfaces, which take the value null at the centre of mass of the spot for $q=1,2, \ldots$ Furthermore, their values in a vicinity of the propagation axis are significantly small. The higher the value of $q$, the bigger the vicinity size. In addition, $\left(x^{2}+y^{2}\right)^{q}=1$ at the edge of the spot support as is shown in Figure 1.

In other words, $V_{P}^{(q)}(x, y ; \omega)$ will increase a specific fraction of $\rho_{P}(x, y ; \omega)$, outside of a vicinity of its centre of mass, as Figure 2 shows. This fraction can be characterised by only the first- and second- order moments of $V_{P}^{(q)}(x, y ; \omega)$, i.e.

$$
\gamma_{j k}^{(q)}=\iint_{P} x^{j} y^{k} V_{P}^{(q)}(x, y ; \omega) d x d y \quad \text { with } j+k=1,2 \text { and } q=0,1,2, \ldots
$$


TABLE 1: Associated density functions up to the fifth order. The denominators are corresponding to the normalization constants $C_{q}^{-1}$

\begin{tabular}{|c|c|c|c|}
\hline$q$ & $V_{P}^{(q)}(x, y ; \omega)$ & $q$ & $V_{p}^{(a)}(x, y ; \omega)$ \\
\hline 0 & $\rho_{P}(x, y ; \omega)$ & 3 & $\frac{\left(x^{6}+3 x^{4} y^{2}+3 x^{2} y^{4}+y^{6}\right)}{\gamma_{60}+3 \gamma_{42}+3 \gamma_{24}+\gamma_{06}} \rho_{P}(x, y ; \omega)$ \\
\hline 1 & $\frac{\left(x^{2}+y^{2}\right)}{\gamma_{20}+\gamma_{02}} \rho_{P}(x, y ; \omega)$ & 4 & $\frac{\left(x^{8}+4 x^{6} y^{2}+6 x^{4} y^{4}+4 x^{2} y^{6}+y^{8}\right)}{\gamma_{80}+4 \gamma_{62}+6 \gamma_{44}+4 \gamma_{26}+\gamma_{08}} \rho_{P}(x, y ; \omega)$ \\
\hline 2 & $\frac{\left(x^{4}+2 x^{2} y^{2}+y^{4}\right)}{\gamma_{40}+2 \gamma_{22}+\gamma_{04}} \rho_{P}(x, y ; \omega)$ & 5 & $\frac{\left(x^{10}+5 x^{8} y^{2}+10 x^{6} y^{4}+10 x^{4} y^{6}+5 x^{2} y^{8}+y^{10}\right)}{\gamma_{10,0}+5 \gamma_{82}+10 \gamma_{64}+10 \gamma_{46}+5 \gamma_{28}+\gamma_{0,10}} \rho_{P}(x, y ; \omega)$ \\
\hline
\end{tabular}
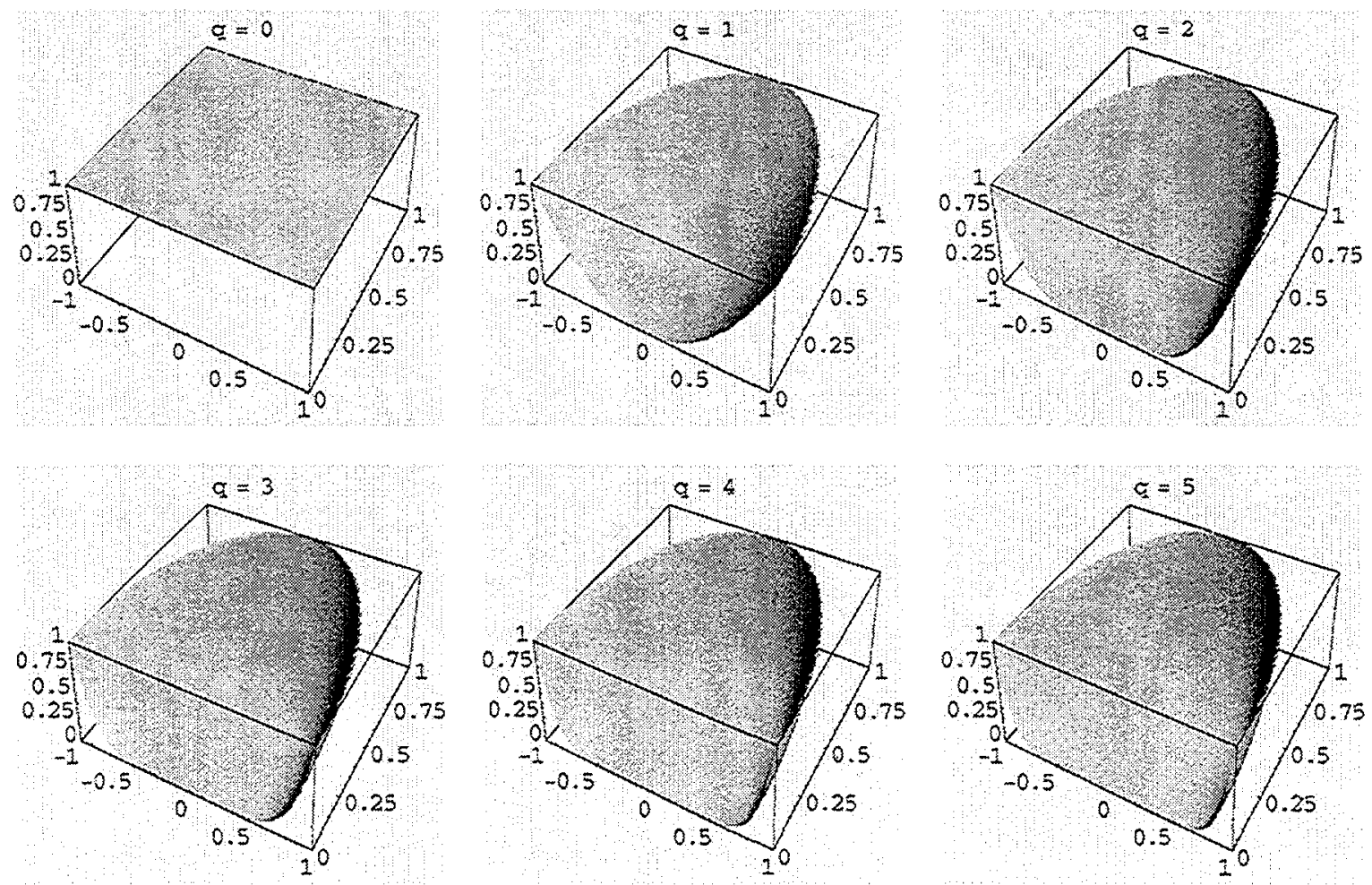

Fig. 1: $\left(x^{2}+y^{2}\right)^{a}$ for $q=0,1, \ldots 5$. These functions are revolution surfaces but only its section for $0 \leq y \leq 1$ is shown to appreciate their features in the vicinity of the coordinates origin. 



Fig. 2: $V_{p}^{(\tau)}(x, y ; \omega)$, with $q=0,1, \ldots .5$, for a rotation symmetric (Gaussian) spot.
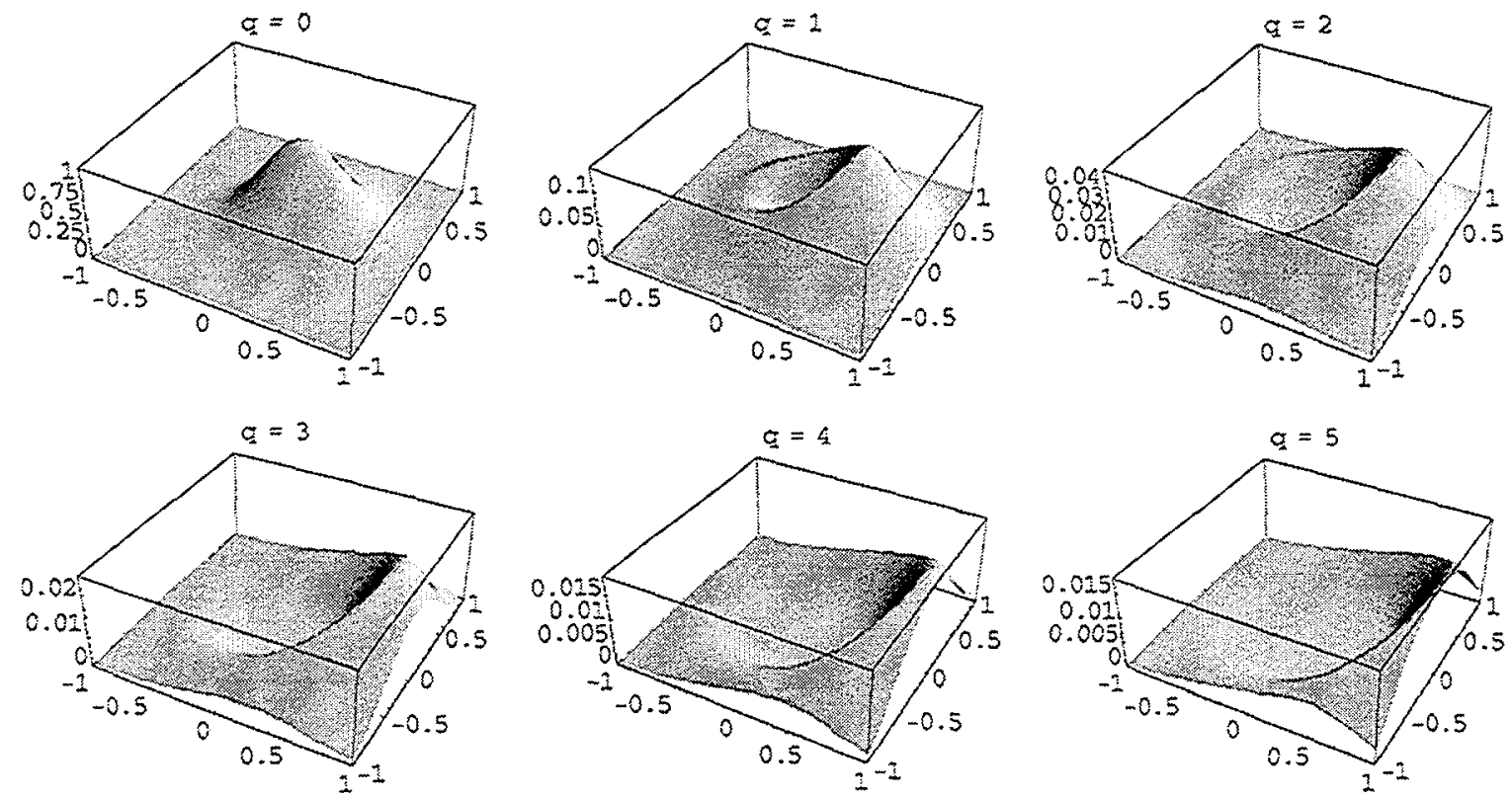

Fig. 3: $V_{P}^{(q)}(x, y ; \omega)$, with $q=0,1, \ldots 5$, for a non-rotation symmetric spot.

The moments $\gamma_{j k}^{(q)}$ are interpreted in the usual fashion, that is, $\gamma_{00}^{(q)}=1$ denotes the normalization of $V_{P}^{(q)}(x, y ; \omega),\left(\gamma_{10}^{(q)}, \gamma_{01}^{(q)}\right)$ will be the coordinates of its centre of mass, and $\gamma_{20}^{\prime(q)}$ and $\gamma_{02}^{(q)}$ will define its principal axes after the rotation given by the condition $\gamma_{11}^{(q)}=0$, i.e. by the angle in equation (4). 
But the most important property of the moments $\gamma_{j k}^{(q)}$ is that they can be expressed as linear combinations of higher order moments $\gamma_{n m}$, as exemplified in Table 2. Consequently, if the beam spot exhibits even-symmetry with respect to its centre of mass (i.e. it is rotation symmetric), all its odd-order moments will be equal to null and $\gamma_{j k}=\gamma_{k j}$ for $j, k=0,2,4, \ldots$ It means that $\gamma_{10}^{(q)}=\gamma_{01}^{(q)}=\gamma_{11}^{(q)}=0$ and $\gamma_{20}^{(q)}=\gamma_{02}^{(q)}$ for $q=0,1,2, \ldots$

TABLE 2: First- and second-order moments of the associated density functions

\begin{tabular}{|c|c|c|}
\hline$q$ & $\gamma_{10}^{(q)}$ & $\gamma_{01}^{(q)}$ \\
\hline 0 & 0 & 0 \\
\hline 1 & $\frac{\gamma_{30}+\gamma_{12}}{\gamma_{20}+\gamma_{02}}$ & $\frac{\gamma_{21}+\gamma_{03}}{\gamma_{20}+\gamma_{02}}$ \\
\hline 2 & $\frac{\gamma_{50}+2 \gamma_{32}+\gamma_{14}}{\gamma_{40}+2 \gamma_{22}+\gamma_{04}}$ & $\frac{\gamma_{41}+2 \gamma_{23}+\gamma_{05}}{\gamma_{40}+2 \gamma_{22}+\gamma_{04}}$ \\
\hline 3 & $\frac{\gamma_{70}+3 \gamma_{52}+3 \gamma_{34}+\gamma_{16}}{\gamma_{60}+3 \gamma_{42}+3 \gamma_{24}+\gamma_{06}}$ & $\frac{\gamma_{61}+3 \gamma_{43}+3 \gamma_{25}+\gamma_{07}}{\gamma_{60}+3 \gamma_{42}+3 \gamma_{24}+\gamma_{06}}$ \\
\hline 4 & $\frac{\gamma_{90}+4 \gamma_{12}+6 \gamma_{54}+4 \gamma_{36}+\gamma_{18}}{\gamma_{80}+4 \gamma_{62}+6 \gamma_{44}+4 \gamma_{26}+\gamma_{08}}$ & $\frac{\gamma_{81}+4 \gamma_{63}+6 \gamma_{45}+4 \gamma_{27}+\gamma_{09}}{\gamma_{80}+84 \gamma_{62}+6 \gamma_{44}+4 \gamma_{26}+\gamma_{08}}$ \\
\hline 5 & $\frac{\gamma_{11,0}+5 \gamma_{92}+10 \gamma_{74}+10 \gamma_{56}+5 \gamma_{38}+\gamma_{1,10}}{\gamma_{10,0}+5 \gamma_{82}+10 \gamma_{64}+10 \gamma_{46}+5 \gamma_{28}+\gamma_{0,10}}$ & $\frac{\gamma_{10,1}+5 \gamma_{83}+10 \gamma_{65}+10 \gamma_{47}+5 \gamma_{29}+\gamma_{0,11}}{\gamma_{10,0}+5 \gamma_{82}+10 \gamma_{64}+10 \gamma_{46}+5 \gamma_{28}+\gamma_{0,10}}$ \\
\hline
\end{tabular}

\begin{tabular}{|c|c|c|c|}
\hline$q$ & $\gamma_{20}^{(q)}$ & $\gamma_{11}^{(q)}$ & $\gamma_{02}^{(q)}$ \\
\hline 0 & $\gamma_{20}$ & $\gamma_{11}$ & $\gamma_{02}$ \\
\hline 1 & $\frac{\gamma_{40}+\gamma_{22}}{\gamma_{20}+\gamma_{12}}$ & $\frac{\gamma_{31}+\gamma_{13}}{\gamma_{20}+\gamma_{02}}$ & $\frac{\gamma_{22}+\gamma_{04}}{\gamma_{20}+\gamma_{02}}$ \\
\hline 2 & $\frac{\gamma_{60}+2 \gamma_{42}+\gamma_{24}}{\gamma_{40}+2 \gamma_{22}+\gamma_{04}}$ & $\frac{\gamma_{51}+2 \gamma_{33}+\gamma_{15}}{\gamma_{40}+2 \gamma_{22}+\gamma_{04}}$ & $\frac{\gamma_{42}+2 \gamma_{24}+\gamma_{06}}{\gamma_{40}+2 \gamma_{22}+\gamma_{04}}$ \\
\hline 3 & $\frac{\gamma_{80}+3 \gamma_{62}+3 \gamma_{44}+\gamma_{26}}{\gamma_{60}+3 \gamma_{42}+3 \gamma_{24}+\gamma_{06}}$ & $\frac{\gamma_{71}+3 \gamma_{53}+3 \gamma_{35}+\gamma_{16}}{\gamma_{60}+3 \gamma_{42}+3 \gamma_{24}+\gamma_{06}}$ & $\frac{\gamma_{62}+3 \gamma_{44}+3 \gamma_{26}+\gamma_{03}}{\gamma_{60}+3 \gamma_{42}+3 \gamma_{24}+\gamma_{06}}$ \\
\hline 4 & $\frac{\gamma_{10.0}+4 \gamma_{82}+6 \gamma_{64}+4 \gamma_{46}+\gamma_{28}}{\gamma_{80}+4 \gamma_{62}+6 \gamma_{44}+4 \gamma_{26}+\gamma_{08}}$ & $\frac{\gamma_{91}+4 \gamma_{73}+6 \gamma_{55}+4 \gamma_{37}+\gamma_{19}}{\gamma_{80}+4 \gamma_{62}+6 \gamma_{44}+4 \gamma_{26}+\gamma_{08}}$ & $\frac{\gamma_{82}+4 \gamma_{64}+6 \gamma_{46}+4 \gamma_{28}+\gamma_{0,10}}{\gamma_{80}+4 \gamma_{62}+6 \gamma_{44}+4 \gamma_{26}+\gamma_{08}}$ \\
\hline 5 & $\frac{\gamma_{12,0}+5 \gamma_{10,2}+10 \gamma_{84}+10 \gamma_{66}+5 \gamma_{48}+\gamma_{2,10}}{\gamma_{10,0}+5 \gamma_{82}+10 \gamma_{64}+10 \gamma_{46}+5 \gamma_{28}+\gamma_{0,10}}$ & $\frac{\gamma_{11,1}+5 \gamma_{93}+10 \gamma_{75}+10 \gamma_{57}+5 \gamma_{39}+\gamma_{1,11}}{\gamma_{10,0}+5 \gamma_{82}+10 \gamma_{64}+10 \gamma_{46}+5 \gamma_{28}+\gamma_{0,10}}$ & $\frac{\gamma_{10,2}+5 \gamma_{84}+10 \gamma_{66}+10 \gamma_{48}+5 \gamma_{2,10}+\gamma_{0,12}}{\gamma_{10,0}+5 \gamma_{82}+10 \gamma_{64}+10 \gamma_{46}+5 \gamma_{28}+\gamma_{0,10}}$ \\
\hline
\end{tabular}

Differently expressed, all the associated density functions will exhibit even symmetry (i.e. they will be rotation symmetric, concentric and their principal axes will be respectively parallel and parallel to the coordinate axes) as shown in Figure 2.

Asymmetries in the spot deal to non-null values for $\gamma_{10}^{(q)}, \gamma_{01}^{(g)}$ and/or $\gamma_{11}^{(g)}$. Shifts of the centres of mass of the associated density functions with respect to the centre of mass of the spot will be revealed by non-null values of $\gamma_{10}^{(q)}$ and/or $\gamma_{01}^{(q)}$. Non-null eccentricities will be given if $\gamma_{20}^{(q)} \neq \gamma_{02}^{(q)}$ and axes rotations if $\gamma_{11}^{(q)} \neq 0$. Figure 3 shows the associated density function for a non-rotation symmetric spot. 
The vector $\left\langle\mathbf{r}^{(q)}\right\rangle \leftrightarrow\left(\gamma_{10}^{(q)}, \gamma_{01}^{(q)}\right)$ denotes the shift of the centre of mass of $V_{P}^{(q)}(x, y ; \omega)$ with respect to the centre of mass of $\rho_{P}(x, y ; \omega)$. As customary, we regard a shift smaller than the $20 \%$ of the mean radius of the spot as permissible. So, if $\left|\left\langle\mathbf{r}^{(q)}\right\rangle\right|=\sqrt{\left(\gamma_{10}^{(q)}\right)^{2}+\left(\gamma_{01}^{(q)}\right)^{2}}>0.2\left(\frac{a^{(0)}+b^{(0)}}{2}\right)$ then the beam spot will be coma-like distorted by the shift of the centre of mass of $V_{P}^{(q)}(x, y ; \omega)$.

The lengths of the principal axes of the $V_{P}^{(q)}(x, y ; \omega)$ will be given by $a^{(q)}=\sqrt{\gamma_{20}^{\prime(q)}}$ and $b^{(q)}=\sqrt{\gamma_{02}^{\prime(q)}}$, with the primes denoting the values of the moments after the rotation of the coordinate axes by the angle given by equation (4), in such a way that the condition $\gamma_{11}^{(q)}=0$ holds. Thus its eccentricity will be given by $E_{P}^{(q)}=\sqrt{1-\left(\frac{a^{(q)}}{b^{(q)}}\right)^{2}}$ provided $a^{(q)} \leq b^{(q)}$. By applying the same criterion as before, even symmetric (i.e. stigmatic) $V_{P}^{(q)}(x, y ; \omega)$ will be obtained for $0 \leq E_{P}^{(q)} \leq 0.3$ and astigmatic ones for $E_{P}^{(q)}>0.3$.

On the other hand, it is important to note that the moments $\gamma_{j k}^{(q)}$ for a given $q$ only depend on specific sets of moments $\gamma_{m n}$. Except for the constant $C_{q}$, the first-order moments $\gamma_{10}^{(q)}$ and $\gamma_{01}^{(q)}$ will depend on the $\gamma$-moments of order $2 q+1$, and the second-order ones $\gamma_{20}^{(q)}, \gamma_{11}^{(q)}$ and $\gamma_{02}^{(q)}$ will depend on the $\gamma$-moments of order $2(q+1)$. It means that the fraction of the beam spot enhanced by each associated density function will be analyzed by using separated sets of higher-order moments. In this sense, different $V_{p}^{(q)}(x, y ; \omega)$ will be characterised by independent descriptors.

A further important descriptor is the power fraction corresponding to each associated density function. Specifically, the power fraction for $V_{P}^{(q)}(x, y ; \omega)$ will be contained within the elliptical crown delimited by the main axes $\left(a^{(q-1)}, b^{(q-1)}\right)$ and $\left(a^{(q)}, b^{(q)}\right)$, with $\left(a^{(-1)}, b^{(-1)}\right) \equiv(0,0)$ for $q=0$, as shown in Figure 4. Thus the power percent will be given by

$$
S_{P}^{(q)}(\omega)=\frac{\iint_{P} S_{P}(x, y ; \omega) d x d y}{\iint_{P} S_{P}(x, y ; \omega) d x d y} .
$$




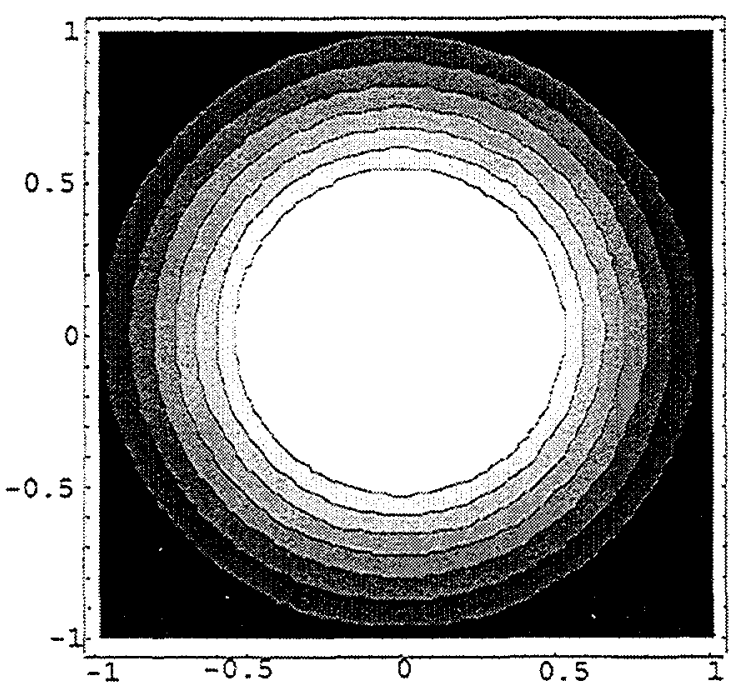

a

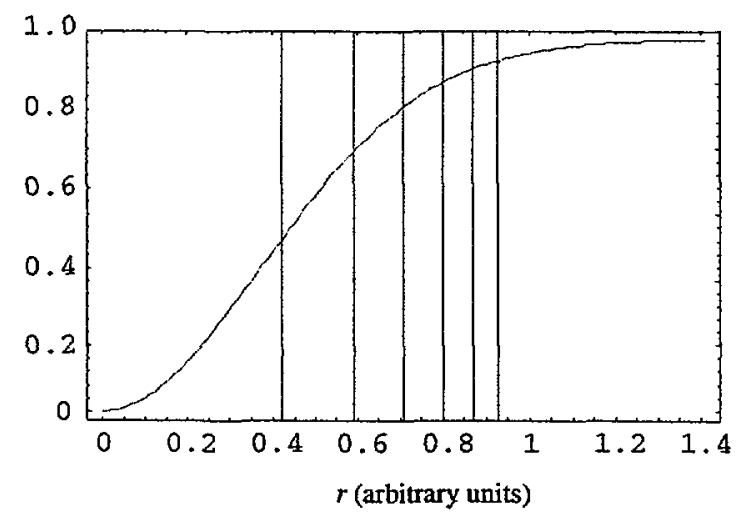

b

Fig. 4: Power fractions for the associated density functions of a rotation symmetric (Gaussian) spot. The circumferences in a) are corresponding to each $V_{P}^{(q)}(x, y ; \omega)$. The curve in $\left.\mathrm{b}\right)$ is the power contained within a circumference of radius $r$. The maximum power is normalized to 1 . The vertical lines are corresponding to the radii of the circumferences in a).

The spot analysis can be considered complete within an error of 5\% when the power fraction contained within the most external ellipse is at least the $95 \%$ of the total power. The associated function corresponding to this ellipse will determine the highest-order moments which are needed to completely characterize the spot.

\section{SIMULATION RESULTS}

For the simulations we have considered two spots, i.e. one of them rotation symmetrical (Gaussian) and the other asymmetrical as depicted in Figures 2 and 3 for $q=0$. Two features of recorded spots are crucial for calculating the centred reduce moments accurately, and should be properly treated before to perform it. They are the noise suppression and the scaling of the spot to adequately match the integration domain, i.e. the domain of the $\left(x^{2}+y^{2}\right)^{q}$ functions.

Usually, spots are recorded in presence of low power additive noise, mainly thermal noise of the detector, as exemplified in Figure 5 for the Gaussian spot. Although information about spot features is still recognizable in $V_{P}^{(1)}(x, y ; \omega)$, its moments $\gamma_{j k}^{(1)}$ should be strong influenced by the enhanced noise. Now, the higher the value of $q$ the stronger the noise enhancement, so that for $q>1$ noise will be predominant over the spot information in $V_{p}^{(q)}(x, y ; \omega)$. Consequently, the values of the moments $\gamma_{j k}^{(q)}$ will mainly refer to the noise distribution.

Therefore, noise suppression must be performed prior to the calculation of the moments. This type of background noise can be effectively suppressed by conventional histogram clipping and offset restore as explaining in References 12 and 13. The results are shown in Fig. 2. 



Fig. 5: Background noise effects on the associated density functions of a rotation symmetric (Gaussian) spot. Because of them, higher order moments cannot give information about the spot properties

Once the noise is suppressed, the spot can be properly scaled into the region $[-1,1] \times[-1,1]$. For very narrow spots only the lower order associated density functions will have significant values. Consequently, detailed information of the spot structure will be not reachable. On the contrary, if the spot support is greater than the region $[-1,1] \times[-1,1]$, complete information of the spot structure cannot be obtained although the associated density functions take significant values.

The encircled energy curve [14] can be used to properly scale the spot support onto the region $[-1,1] \times[-1,1]$. Each point of the curve is corresponding to the power fraction within a square with side $0<d \leq D$, with $D$ the side of the spot support. Thus, the curve can be depicted on a two dimensional graph of power content vs diagonal of the square. Consequently, the basis of the square is obtained by dividing the abscise values by $\sqrt{2}$. The curve growths monotonically and approaches asymptotically to a constant value, as shown in Figure 6.

For spots that spread out the region $[-1,1] \times[-1,1]$, the curve does not raise the asymptotical approach, whilst jump-like curves are obtained by very narrow spots. An optimal matching will be obtained when the curve ends at the beginning of the asymptotical approach (Figure 6). It can be reasonably estimated from the first derivative of the curve.

Indeed, this derivative exhibits an absolute maximum and a relatively fast decay after the maximum. The beginning of the asymptotical approach of the curve can be regarded at the point where the first derivative has decayed to the $1 \%$ of its maximum value. This point of the domain of the encircled energy curve will determine the side length of the spot support with the best matching to the region $[-1,1] \times[-1,1]$ (Figure 6). 

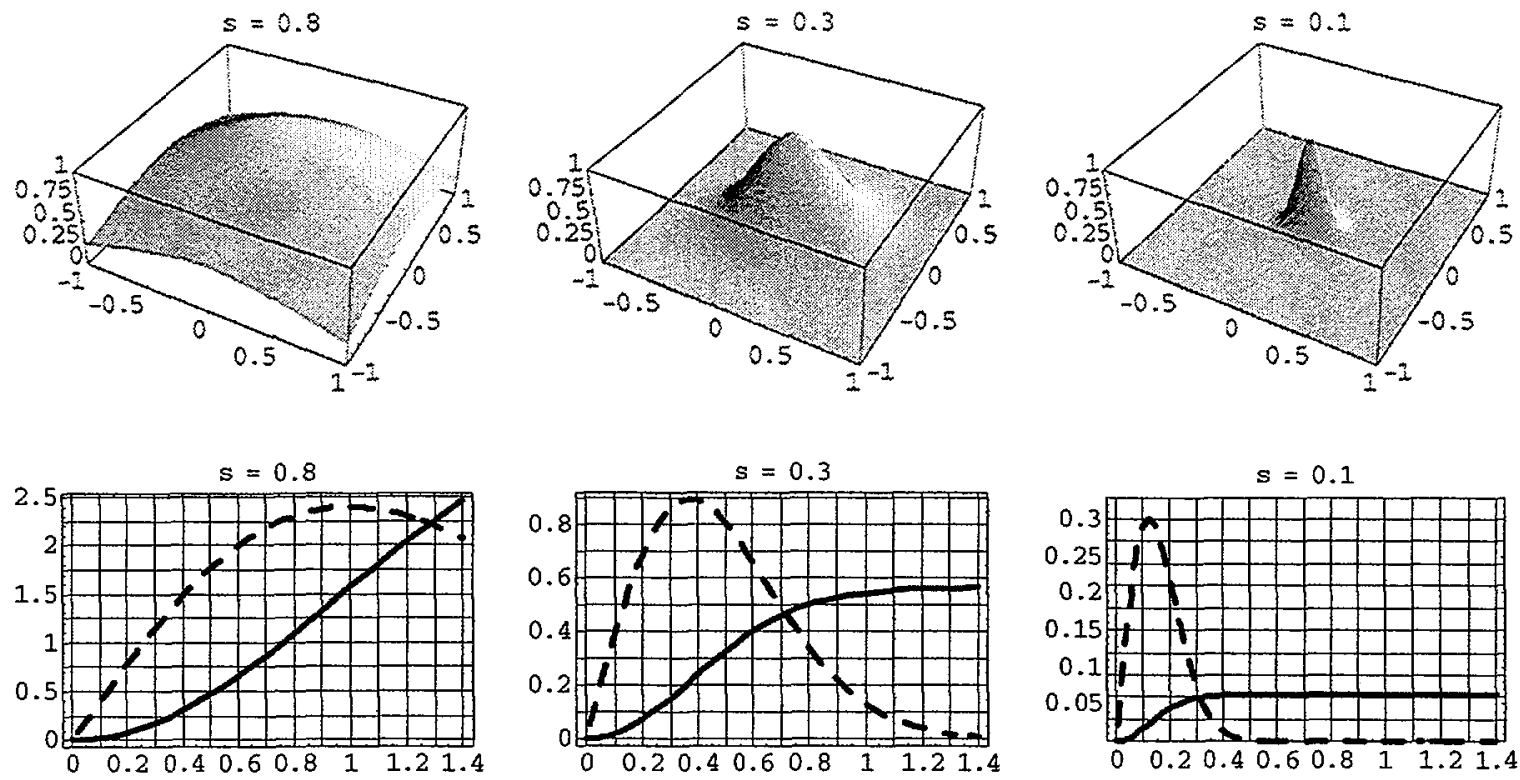

Fig. 6: Matching of the spot support and the region $[-1,1] \times[-1,1]$ for Gaussian spots of standard deviation s. The figure on the left up depicts a spot that spreads out this region. Its encircled energy curve does not raise the asymptotical region (figure on the left bottom). The spot on the right up is to narrow, so that its encircled energy curve is jump-like (figure on the right bottom). The best matching is corresponding to the Gaussian spot on the middle up. Its support was determined by applying the criterion in the text (figure on the middle down). Additionally the dashed lines in plots show the first derivative of the enclosed energy curves with respect to $r$.

After performing the best matching, useful associated density functions can be accurately determined, as illustrated in Figures 2 and 3. Table 3 shows the moments of the associated density functions in these figures.

As expected, $\gamma_{10}^{(0)}=\gamma_{01}^{(0)}=0$ for both spots, and $\gamma_{10}^{(q)}=\gamma_{01}^{(q)}=\gamma_{11}^{(q)}=0$ and $\gamma_{20}^{(q)}=\gamma_{02}^{(q)}$ for the Gaussian spot. So, the centres of mass of all the associated density functions of the Gaussian spot will be located at the coordinate origin, and that their corresponding main axes will be parallel to each other and parallel to the coordinate axes. Furthermore, the condition $\gamma_{20}^{(q)}=\gamma_{02}^{(q)}$ points out that the main axes of each associated density function are of the same length so that the corresponding eccentricity will be equal to null. These results are in complete correspondence to the rotation symmetry of the Gaussian spot.

TABLE 3: First- and second-order moments of the associated density functions in Figures 2 and 3

\begin{tabular}{|c|c|c|c|c|c|c|c|c|c|c|}
\hline \multicolumn{3}{|c|}{ Rotation symmetrical (Gaussian) spot } & \multicolumn{3}{|c|}{ Non-rotation symmetrical spot } \\
\hline$q$ & $\gamma_{10}^{(q)}$ & $\gamma_{01}^{(q)}$ & $\gamma_{20}^{(q)}$ & $\gamma_{11}^{(q)}$ & $\gamma_{02}^{(q)}$ & $\gamma_{10}^{(q)}$ & $\gamma_{01}^{(q)}$ & $\gamma_{20}^{(q)}$ & $\gamma_{11}^{(q)}$ & $\gamma_{02}^{(q)}$ \\
\hline 0 & 0 & 0 & 0.0891 & 0 & 0.0891 & 0 & 0 & 0.0883 & 0 & 0.0891 \\
\hline 1 & 0 & 0 & 0.1743 & 0 & 0.1743 & 0.2480 & 0 & 0.1488 & 0 & 0.1670 \\
\hline 2 & 0 & 0 & 0.2521 & 0 & 0.2521 & 0.3425 & 0 & 0.1874 & 0 & 0.2337 \\
\hline 3 & 0 & 0 & 0.3202 & 0 & 0.3202 & 0.4179 & 0 & 0.2104 & 0 & 0.2910 \\
\hline 4 & 0 & 0 & 0.3790 & 0 & 0.3790 & 0.4776 & 0 & 0.2233 & 0 & 0.3418 \\
\hline 5 & 0 & 0 & 0.4303 & 0 & 0.4303 & 0.5258 & 0 & 0.2298 & 0 & 0.3891 \\
\hline
\end{tabular}


For the non-rotation symmetrical spot there is a shifting of the centres of mass of the associated density functions with respect to the centre of mass of the spot along the $x$-axis, because $\gamma_{10}^{(q)} \neq 0$ and $\gamma_{01}^{(q)}=0$ for $q \geq 1$. Furthermore, the principal axes will be not rotated because $\gamma_{11}^{(q)}=0$, although eccentricities bigger than null should be determined because $\gamma_{20}^{(q)}>\gamma_{02}^{(q)}$, i.e. $a^{(q)}>b^{(q)}$. It means that this spot should be an approximately elliptical support with its major principal axis parallel to the $x$-axis.

The above analyse are confirmed by the spot descriptors in Table 4 . The values of all the descriptors are smaller than one because they are referred to the dimensionless region $[-1,1] \times[-1,1]$. Scale factors are needed to express them in the appropriate units in practical applications.

TABLE 4: Descriptors of the associated density functions in Figures 2 and 3

\begin{tabular}{|c|c|c|c|c|c|c|c|c|c|c|}
\hline & \multicolumn{5}{|c|}{ Rotation symmetrical (Gaussian) spot } & \multicolumn{5}{|c|}{ Non-rotation symmetrical spot } \\
\hline$q$ & $\begin{array}{c}\text { C- } \\
\text { Vecto } \\
\mathbf{r}\end{array}$ & $\begin{array}{l}\text { Main } \\
\text { axis } a\end{array}$ & $\begin{array}{l}\text { Main } \\
\text { axis } b\end{array}$ & $\begin{array}{c}\text { Ecce } \\
\text { ntric } \\
\text { ity }\end{array}$ & $\begin{array}{c}\text { Encirc } \\
\text { led } \\
\text { energy }\end{array}$ & $\begin{array}{c}\text { C- } \\
\text { Vector }\end{array}$ & $\begin{array}{l}\text { Main } \\
\text { axis } a\end{array}$ & $\begin{array}{l}\text { Main } \\
\text { axis } b\end{array}$ & $\begin{array}{c}\text { Ecce } \\
\text { ntrici } \\
\text { ty }\end{array}$ & $\begin{array}{l}\text { Encircl } \\
\text { ed } \\
\text { energy }\end{array}$ \\
\hline 0 & $(0,0)$ & 0.2985 & 0.2985 & $\mathbf{0}$ & 0.4671 & $(\mathbf{0}, \mathbf{0})$ & $\begin{array}{c}0.298 \\
5\end{array}$ & 0.2971 & $\mathbf{0}$ & 0.4657 \\
\hline 1 & $(0,0)$ & 0.4175 & 0.4175 & $\mathbf{0}$ & 0.7057 & $\begin{array}{c}(0.2480 \\
, 0)\end{array}$ & $\begin{array}{c}0.408 \\
6\end{array}$ & 0.3858 & $\begin{array}{c}0.329 \\
7\end{array}$ & 0.6017 \\
\hline 2 & $(0,0)$ & 0.5021 & 0.5021 & $\mathbf{0}$ & 0.8286 & $\begin{array}{c}(0.3425 \\
, 0)\end{array}$ & $\begin{array}{c}0.483 \\
4\end{array}$ & 0.4330 & $\begin{array}{c}0.444 \\
8\end{array}$ & 0.7711 \\
\hline 3 & $(0,0)$ & 0.5659 & 0.5659 & $\mathbf{0}$ & 0.8936 & $\begin{array}{c}(0.4179 \\
, 0)\end{array}$ & $\begin{array}{c}0.539 \\
4 \\
\end{array}$ & 0.4588 & $\begin{array}{c}0.526 \\
1\end{array}$ & 0.8268 \\
\hline 4 & $(0,0)$ & 0.6156 & 0.6156 & 0 & 0.9304 & $\begin{array}{c}(0.4776 \\
, 0)\end{array}$ & $\begin{array}{c}0.584 \\
7\end{array}$ & 0.4725 & $\begin{array}{c}0.588 \\
9\end{array}$ & 0.8614 \\
\hline 5 & $(0,0)$ & 0.6560 & 0.6560 & $\mathbf{0}$ & 0.9525 & $\begin{array}{c}(0.5258 \\
, 0)\end{array}$ & $\begin{array}{c}0.623 \\
8\end{array}$ & 0.4793 & $\begin{array}{c}0.640 \\
0\end{array}$ & 0.8852 \\
\hline
\end{tabular}

Taking into account that the total energy content is normalized to 1 , the encircled energy values can be interpreted as percent values. According to the asymptotic behaviour of the encircled energy, the hihgher the value of $q$ the smaller the value of its increment. Furthermore, according to the regarded error (5\%) in the encircled energy, the analysis of the Gaussian spot can be considered complete. However, an additional associated density function $(q=6)$ should be considered to complete the analysis of the non-rotation symmetrical spot, because up to $q=5$ only $88.5 \%$ of the total energy can be analyzed.

The pairs of numbers in parenthesis denote the $(x, y)$ components of the C-Vectors, which describe the shifting of the centre of mass of each associated density function with respect to the centre of mass of the spot density. As expected, all the C-Vectors will be of null length for the Gaussian spot, whilst for the non-rotation symmetrical spot, they will have values bigger than null.

Now, according to the condition we regarded before, if the length of the C-Vector is greater than 0.05956 , the non-rotation symmetrical spot should be coma-like distorted. It is the case for all $V_{P}^{(q)}(x, y ; \omega)$ with $q \geq 1$, which exhibits in general a strong distortion. However, although the associated density functions are progressively distorted, the more significant coma-like distortion occurs for $q=1$. Indeed, about $60 \%$ of the total energy is mainly affected for a C-Vector of length 
0.2480 . The distortion for $q=2$ will be corresponding to a length increase of 0.0945 of the C-Vector and affects the additional encircled energy, i.e. $16.94 \%$ of the total energy. For $q=3$ the length increase of the C-Vector is 0.0754 . It affects an additional encircled energy of $5.57 \%$, on so on.

As expected for the Gaussian spot, all the eccentricities are equal to null, i.e. this spot is stigmatic. For the non-rotation symmetrical spot, the eccentricities are bigger than 0.3 for $q \geq 1$. That means that this spot is astigmatic. Nevertheless, it should be a non-severe astigmatism because about $60 \%$ of the total energy is distributed onto an ellipse, whose principal axes only differ in $5.58 \%$. This distortion increases significantly with the value of $q$, but affects smaller additional encircled energy.

Summarizing, the non-rotation symmetrical spot we analysed as example exhibits a relative strong coma-like distortion but a relative soft astigmatism-like distortion.

\section{CONCLUSION}

We have shown a method for exhaustively analyzing beam spots. It is based on the central reduced moments of the spot. Specific combinations of higher order moments can be interpreted as center of mass coordinates and length and orientations of the principal axis of associated density functions.

These functions allow us to separately analyze specific spot fractions. So, the descriptors of the associated density functions deal to the quantitative estimation of spot features, such coma-like and astigmatism-like distortions for example.

To assure high accuracy, background noise suppression and an optimal match of the spot support onto the region $[-1,1] \times[-1,1]$ must be performed prior to the calculation of the moments.

\section{Acknowledgments}

This work was finished during a visit of Roman Castaneda to the Abdus Salam International Centre for Theoretical Physics (AS-ICTP), Trieste, Italy, in February 2003. The authors thank Colciencias (Grant 1118-05-11518), and the AS-ICTP for the financial support.

\section{REFERENCES}

1. Dragoman, D. The Wigner distribution function in optics and optoelectronics. Progress in Optics 37, Emil Wolf ed. Elsevier, Amsterdam, 1997. 1-56.

2. Simon, $\mathrm{R}$. and N. Mukunda. Optical phase space, Wigner representation and invariant quality parameters. J. Opt. Soc. Am. A 17 (2000) 2440-2463.

3. Bastiaans, M.J. Application of the Wigner distribution function to partially coherent light. J. Opt. Soc. Am. A 3 (1986) 1227-1238.

4. Martinez-Herrero, M., P.M. Mejias, S. Bosch and A. Carnicer. Spatial width and power-content ratio of hard-edge diffracted beams. J. Opt. Soc. Am. A 20 (2003) 388-391.

5. Sheppard, C.J.R. and Larkin, K.G. Focal shift, optical transfer function and phase space representations. J. Opt. Soc. Am. A 17 (2000) 772-779.

6. Wolf, K.B., M.A. Alonso and G.W. Forbes. Wigner functions for Helmholtz wavefields. J. Opt. Soc. Am. A 16 (1999) 2476-2487.

7. Yang, J. and Fan, D. Intensity-moments characterisation of general pulsed paraxial beams with the Wigner distribution function. J. Opt. Soc. Am. A 16 (1999) 2488-2493.

8. Dragoman, D. Higher-order moments of the Wigner distribution functions in first-order optical systems. J. Opt. Soc. Am. A 11 (1994) 2643-2646. 
9. Castañeda, R. and J. García. Spatial coherence wavelets: mathematical properties and physical features. Accepted for publication in Journal of Modern Optics, 2003.

10. Castañeda, R. and J. García. Spatial coherence wavelets. In press in Journal of Modern Optics, 2002.

11. Castañeda, R. y J.C. Correa. A simple method for analyzing the tails of distributions. Revista de la Academia Colombiana de Ciencias Exactas, Físicas y Naturales. Vol. XXIII, No. 89, Dic. 1999. 555-562.

12. Castañeda, R. Imaging quality evaluation of rotation symmetrical systems in the spatial frequency domain. Pure Appl. Opt. 5 (1996) 45-53.

13. Castañeda, R. Two dimensional evaluation of point spread functions using centred reduced moments. Pure Appl. Opt. 3 (1994) 737-755.

14. Born, $M$ and E. Wolf. Principles of Optics. 5. ed. Pergamon Press, Oxford, 1975. 